(C) 2012 IEEE. Personal use of this material is permitted. Permission from IEEE must be obtained for all other uses, in any current or future media, including reprinting/republishing this material for advertising or promotional purposes, creating new collective works, for resale or redistribution to servers or lists, or reuse of any copyrighted component of this work in other works. 


\title{
The NAT64/DNS64 Tool Suite for IPv6 Transition
}

\author{
Marcelo Bagnulo and Alberto García-Martínez, Universidad Carlos III de Madrid \\ Iljitsch van Beijnum, Institute IMDEA Networks and Universidad Carlos III de Madrid
}

\begin{abstract}
It is clear that there is not enough time to upgrade existing Internet hosts to dual stack before the IPv4 address pool depletes. This implies that the IPv6 transition and co-existence must support interaction between IPv4 nodes and IPv6 nodes. In this article we describe NAT64 and DNS64, a tool suite that provides a way forward in the IPv4-to-IPv6 transition by allowing communication among unmodified IPv6 and IPv4 nodes.
\end{abstract}

\section{INTRODUCTION}

IN the early 1990s it became apparent that the 32-bit address size of the IPv4 protocol would be too small in the long run, so the IETF started work on an updated version of IP with larger addresses, IPv6. Unfortunately, the resulting protocol is not backward compatible with IPv4, so there is a need for tools to allow the transition from IPv4 to IPv6 and the coexistence of the two protocols. The IETF created three main transition mechanisms between the existing IPv4 and the new IPv6: tunneling, dual stack, and translation. Tunneling consists of encapsulating an IPv6 packet inside an IPv4 packet to communicate IPv6 nodes across IPv4-only paths. Dual stack means running both IPv4 and IPv6 at the same time in endpoints and in the network, by having dual stack operating systems and applications in upgraded nodes. Dual stack also means having dual stack network equipment, and configuring both types of addresses for new networks. Dual stack was conceived to introduce IPv6 without resigning from IPv4 until all hosts had transitioned and IPv4 could be removed without disruption. However, this transition strategy is no longer viable, as the IANA IPv4 address pool was exhausted on February 3rd of $2011^{1}$ and currently less than 10 percent of the Internet hosts are IPv6 enabled. ${ }^{2}$ The result is that there is no time left to migrate the current installed base of IPv4-only hosts to dual stack before IPv6-only hosts are deployed. This implies the need in the short to medium term for the translation of packets between IPv4-only hosts and IPv6-only hosts to support communication between them.

Stateless IP and ICMP translation (SIIT) [1] and Network Address Translation - Protocol Translation (NAT-PT) [2] specified how to translate between IPv4 and IPv6. SIIT specifies stateless translation, which happens on a perpacket basis without the need of any state. The limitation of stateless translation is the need to maintain a one-to-one mapping between IPv4 and IPv6 addresses. Therefore, the number of IPv6 hosts that can be served by a translator is limited to the number of IPv4 addresses available to the translation service.

NAT-PT provided a stateful mechanism for address translation in which a single IPv4 address could be used to map multiple IPv6 hosts. In order to map the IPv4 destination address back to the correct IPv6 destination address in the IPv4-to-IPv6 direction, the NAT-PT translator maintained temporary state in a translation table. This procedure is similar to the Network Address Translation (NAT) that is popular in IPv4 networks. Due to the larger address space of IPv6, it is easy to initiate communications in this realm to the IPv4 side by embedding the IPv4 destination address into an IPv6 address. However, to enable communications initiated from the IPv4 side, a complex setup involving synchronization between a DNS Application Level Gateway (DNS-ALG) and a Bi-Directional NAT-PT was required. In 2007 the NAT-PT specification was moved to "Historic" status within the IETF after identifying problematic interactions with other aspects of IPv6 operation that resulted in reduced functionality and reliability of the network [3].

NAT64/DNS64 $[4,5]$ is a tool suite intended to replace NAT-PT in a manner that addresses most of the concerns that led to its deprecation. A NAT64 device translates IPv4 packets into IPv6 packets and vice versa in a stateful manner, and DNS64 synthesizes AAAA resource records for domain names that only have A resource records available. A key design decision for NAT64/DNS64 is to explicitly manage only communications initiated from the IPv6 side, while relying on existing NAT-traversal techniques, such as STUN [6], to support peer-to-peer applications. To do so, NAT64 is designed to conform to the requirements and recommendations for translators defined by the IETF BEHAVE working group, thus resulting in consistent behavior of different NAT64 implementations. 


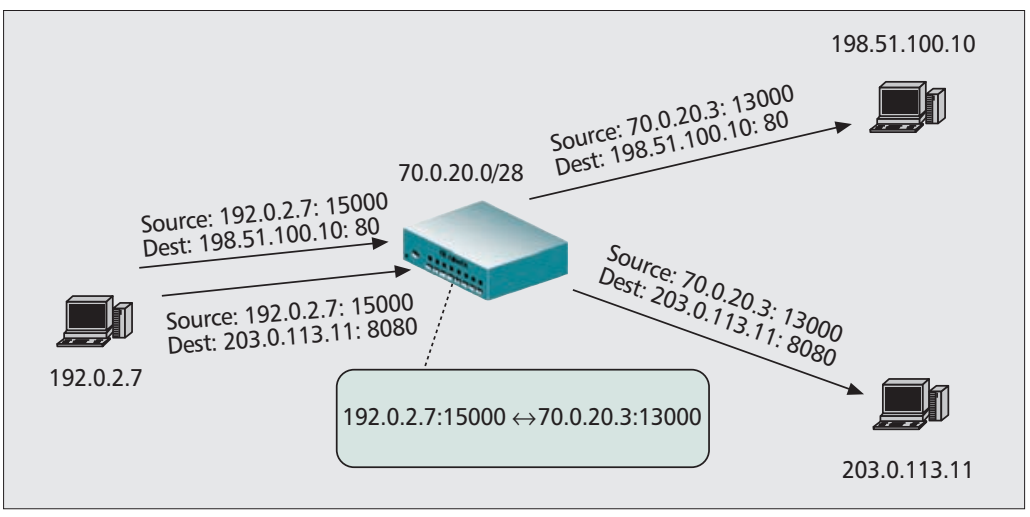

Figure 1. Endpoint independent mapping. guarantee consistent behavior of IPv4-IPv6 translators, a set of requirements should be stated for IPv6-IPv4 translation, analogous to the existing IPv4-IPv4 translation requirements. In order to do that, we take as a starting point the requirements defined for IPv4 NATs. It is straightforward to transpose some of these requirements to the NAT64 context, such as the minimum binding lifetime, port assignment strategies, handling of fragmented packets, etc. However, some other requirements deserve a more careful analysis of its application to the NAT64 case, which is presented next.

In NAT operation it is relevant to distinguish between the mapping behavior and the filtering behavior. In general terms, an IPv4 NAT is a device connecting two realms of IPv4 addressing, one that uses private addresses and another realm that usually is the public Internet. Upon the reception of a packet coming from the private realm, the NAT creates a mapping between the source address and source port pair of the received packet and a public address and port pair available in its own pool. We will refer to an address/port pair as a transport address. The NAT then substitutes the source transport address of the packet with the one assigned in the binding, and forwards the packet to the public realm. The mapping behavior defines how the aforementioned binding is created. Three types of mapping behavior are defined:

Endpoint independent mapping (Fig. 1): The mapping is solely determined by the transport address of the internal host. Packets containing the same private transport address are translated to the same transport address of the NAT's pool irrespectively of their public address and/or port.

Address dependent mapping: The mapping is determined by the transport address of the internal host and the address of the external host. Packets containing the same private transport address and the same public address are translated to the same transport address of the NAT's pool irrespectively of the port used by the external host.

Address and port dependent mapping: The mapping is determined by the transport address of the internal host and the transport address of the external host.

The different types of mappings determine how internal hosts are perceived by external hosts. Different connections initiated by the same process running in a host behind a NAT that uses endpoint independent mappings are presented with the same transport address to external hosts. This is needed by optimized NAT traversal techniques such as STUN [6] and TURN [12]. Both TCP and UDP requirements for NAT operation mandate the use of endpoint independent mappings.

However, this does not imply that a NAT box is required to forward all packets, as filtering rules can apply to comply with security policies. When a NAT box receives a packet through any of its interfaces, it applies the filtering rules to determine whether to forward the packet or to discard it, based on the address and/or port information. The following filtering behaviors are defined:

Endpoint independent filtering: The filtering 
rules only apply to the transport address of the internal host. This means that packets are forwarded or dropped solely based on the transport address of the internal host (either the private one or the one from the NAT's pool assigned through the mapping).

Address dependent filtering: The filtering rules apply to the transport address of the internal host and also on the IP address of the external host.

Address and port dependent filtering: The filtering rules apply to the transport address of the internal host as well as to the transport address of the external host.

The recommendation is for NATs to implement endpoint independent filtering, and if more security is needed, to allow the use of address dependent filtering. While the former type of filtering is compatible with most NAT traversal techniques (including both STUN and TURN), the latter type of filtering is compatible with a reduced set of techniques (it supports TURN but not STUN).

To extend these requirements to the NAT64 case, we need to map the roles of IPv4 and IPv6 addresses to the roles of private and public addresses in IPv4 NATs. Since a mapping can only be created when the translator receives a packet from the IPv6 realm, it is straightforward to map the IPv6 realm in NAT64 to the private realm in IPv4 NATs, and the IPv4 realm in NAT64 to the public realm in IPv4 NATs. The close similarities among the IPv4 NAT and the NAT64 setups allow deriving immediately the mapping and filtering requirements for NAT64, by stating that endpoint independent mappings, and both endpoint independent and address dependent filtering, must be supported.

\section{NAT64}

NAT64 translates IPv6 packets into IPv4 packets and vice versa. It has essentially two components: the address translation mechanism and the protocol translation mechanism. The latter, which translates IP header fields other than the addresses, operates in a stateless manner trying to preserve as much as possible the semantics of the original field. This was originally defined in [1] and has been updated in [13].

Address translation maps IPv6 transport addresses to IPv4 transport addresses and vice versa. In order to create these mappings, the NAT64 box has two pools of IP addresses: an IPv6 address pool (to represent IPv4 addresses in the IPv6 network) and an IPv4 address pool (to represent IPv6 addresses in the IPv4 network).

NAT64 creates the mappings by using an IPv6 prefix (denoted as Pref64::/n) as the IPv6 address pool. Each IPv4 address is mapped into a different IPv6 address by concatenating the Pref64: : / $\mathrm{n}$ with the IPv4 address being mapped and, if $\mathrm{n}$ is less than 96, padding the result to 128 bits with a suffix of zero bits [14]. Pref64:: /n can be either the Well-Known Prefix defined for this purpose (64:ff9b: : /96) [14] or a local prefix manually assigned from the global unicast IPv6 address block of the site for this particular use. In both cases the mapping is
IPv6 derived from well-known prefix

0

\begin{tabular}{|l|l|}
$64: f f 9 b::$ & 198.51 .100 .7 \\
\hline
\end{tabular}

IPv6 derived from local prefix 2001:DB8::/32

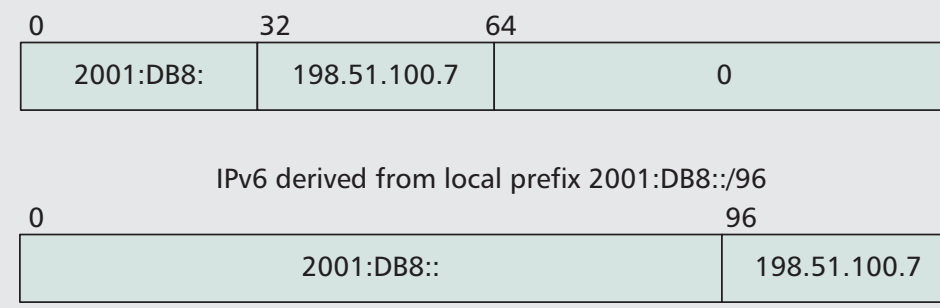

Figure 2. IPv6 address representation for 198.51.100.7.

stable over time since there is no need to re-use the IPv6 addresses, as the IPv6 address space is sufficiently large. By using the Well-Known Prefix, the resulting IPv6 representations of IPv4 addresses are globally meaningful. This allows for any party in the Internet receiving such an address to recognize it as an IPv6 representation of an IPv4 address, and even reach the IPv4 destination if a local NAT64 service is available. This is particularly useful in the case where the DNS64 service and the NAT64 service are supplied by different providers (e.g. using public DNS resolution services). This may be fairly common in mobility scenarios involving multiple domains, where the DNS/DNS64 service is provided by the visited network and the NAT64 service is provided by the home network. Figure 2 shows an example of the representation of an IPv4 address with different IPv6 prefix types.

The IPv4 address pool is normally a small prefix assigned to the NAT64's IPv4 interface. Because of the size of the IPv4 address space relative to the IPv6 address space, the IPv4 address pool is not sufficient to establish permanent one-to-one mappings with IPv6 addresses. So, mappings using the IPv4 address pool are created and released dynamically.

An IPv6 initiator learns the IPv6 address representing the IPv4 target either through the DNS64, as described in the next section, or by other means. Packets to that address sent by the IPv6 host are routed to the NAT64 device. The NAT64 associates an IPv4 transport address from its pool to the IPv6 transport address of the initiator, creating a binding state, so that reply packets can be translated and forwarded back to the initiator. The binding state is kept while packets are flowing. Once packets stop flowing, the binding state times out and the IPv4 transport address is returned to the IPv4 address pool.

In order to implement endpoint-independent mapping and support both endpoint-independent filtering and address-dependent filtering, NAT64 relies on two data structures to store mapping information: the Binding Information Base and the Session table.

The Binding Information Base (BIB) stores only mapping information. Each entry of the BIB corresponds to one transport address of an 
In order to

implement endpoint-

independent map-

ping and support

both endpoint-inde-

pendent filtering and

address-dependent

filtering, NAT64

relies on two data

structures to store

mapping informa-

tion: the Binding

Information Base

and the Session

table.
IPv6 node and the associated IPv4 transport address from the NAT64's IPv4 address pool. When an IPv6 node initiates a new communication using a source transport address that it is not in the BIB, a new entry is created. If the IPv6 node initiates a new communication with an IPv6 transport address for which there is a BIB entry, this entry is reused for this new communication, irrespectively whether the destination IPv6 address or destination port are different from the one used in the previous communications. The result is that multiple communications involving the same IPv6 transport address are translated by the NAT64 to the same IPv4 transport address, resulting in endpoint-independent mapping.

The information contained in the BIB is enough to perform the address translation of any packet and to provide endpoint-independent filtering. However, the information contained in the $\mathrm{BIB}$ is not enough to perform address-dependent filtering. If this is required, the NAT64 needs to keep information about the IPv4 address of the IPv4 node involved in the communication. To support this type of filtering, the NAT64 relies on an additional data structure, the Session table, which contains the source and destination IPv6 transport address as well as the source and destination IPv4 transport address. This allows the NAT64 to verify if an IPv4 packet is addressed to an IPv4 transport address in use from the pool, but also that it comes from an IPv4 address already involved in a communication.

In order to comply with the requirements imposed in the minimum lifetime of the bindings $[9,10]$, each Session table entry has a lifetime, which in the case of UDP is set to 2 minutes, and in the case of TCP is set to 2 hours.

It is apparent that bindings associated to TCP communications are "expensive," in the sense that they consume an IPv4 transport address from the reduced pool for a long time. It thus seems wise to ascertain that there is a real TCP session ongoing before creating the binding. To this end, it is also important to remove the bindings as soon as the NAT64 can determine that the TCP connection is over rather than wait for the binding state to time out. Hence, the NAT64 keeps track of the exchange used to terminate the ongoing TCP connections to remove the associated binding.

In terms of security, the main threats against NAT64 are potential Denial-of-Service attacks, targeted to consume the scarce NAT64 resources including memory, processing power, and the IPv4 transport-address pool. NAT64 can mitigate these attack vectors by limiting the amount of resources assigned to different purposes (e.g. the amount of memory used for temporarily storing fragments waiting for reassembly).

\section{DNS64}

DNS64 synthesizes AAAA resource records (AAAA RRs) from A resource records (A RRs). DNS64 allows IPv6-only hosts to use the Fully Qualified Domain Name of an IPv4-only node to initiate a communication.

When an IPv6-only node starts a communication, it usually queries for a AAAA RR and it expects to obtain the IPv6 address of the target node. To allow an IPv6 initiator to learn the address of the responder, the DNS64 function is used to synthesize a AAAA record from the A record (containing the real IPv4 address of the responder). DNS64 is designed as an additional function of a DNS recursive resolver. As such, when a DNS64 enabled resolver receives a AAAA RR query generated by the IPv6 initiator, it searches for a AAAA RR. If no AAAA record is available for the target node (which would be the case when the target node is an IPv4-only node), the DNS64 performs a query for the A record. If an A record is discovered, the DNS64 creates a synthetic AAAA RR by adding the Pref64:: / $n$ of a NAT64 to the responder's IPv4 address, and if $\mathrm{n}$ is less than 96, a suffix. The synthetic AAAA RR is passed back to the IPv6 initiator, which starts an IPv6 communication with the IPv6 address associated to the IPv4 receiver.

The packet is routed to the NAT64 device, which creates the IPv6-to-IPv4 address mapping as described before. It is important to highlight that the DNS64 and the NAT64 do not share any state. In particular, when the DNS64 generates a synthetic response, no state is created in the NAT64. The only information shared by the NAT64 and the DNS64 is the Pref64::/n, which must be the same for a given domain. By default, both NAT64 and DNS64 are set up to use the Well-Known Prefix, so no manual configuration is required for any of them.

One of the major challenges for DNS64 is the compatibility with DNSSEC. DNSSEC defines extensions to provide origin authentication, authenticated denial of existence, and data integrity of the DNS data. As such, it is in fundamental conflict with DNS64, since DNS64 synthesizes RRs and presents them as RRs originating elsewhere. Unlike the obsolete NATPT DNS-ALG, which intercepted and modified DNS packets, DNS64 is a full-fledged architectural component. Because of that, it is possible to place the DNS64 functionality within the resolution chain of the DNS to be compatible with some modes of DNSSEC by assuring that the synthesis always occurs after validation.

There are different configurations for a recursive resolver involving DNSSEC. A recursive resolver can be DNSSEC-capable or not. Moreover, a DNSSEC-capable resolver can be validating, i.e. performing DNSSEC data validation or not, i.e. simply passing the DNSSEC data.

Next, let's consider how a DNS64 recursive resolver handles different types of DNSSEC queries:

Queries arriving from a non DNSSEC-capable originator (Fig. 3a). A DNSSEC-capable and validating DNS64 recursive resolver can validate the data of the A RR before creating the synthetic AAAA RR.

Queries arriving from a DNSSEC-capable but not validating originator (Fig. $3 b$ ). This is the ideal case for DNS64. If the DNS64 resolver implements DNSSEC validation, it validates the DNSSEC data, creates the synthetic AAAA RR, and signals back to the querying party that the data included is authentic. This is a common case in DNSSEC deployments, where the client 

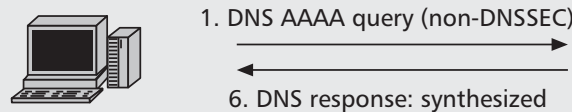

AAAA RR

a) Non-DNSSEC host

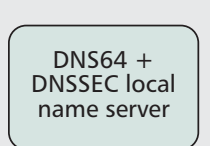

2. DNS AAAA query (DNSSEC, checking)

3. Empty answer in DNS response

4. DNS A query (DNSSEC, checking)

5. DNS response: $A R R+D N S S E C$ data
IPv4 DNSSEC authoritative name server

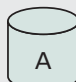

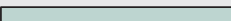

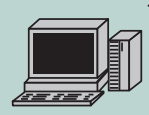

1. DNS AAAA query (DNSSEC, non-checking)

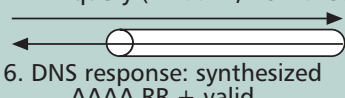

DNS64 +

DNSSEC loca

name server

2. DNS AAAA query (DNSSEC, checking)

3. Empty answer in DNS response

IPV4 DNSSEC

DNS A query (DNSSEC, checking)

AAAA RR + valid

5. DNS response: A RR + DNSSEC data

b) Non-validating DNSSEC-capable host
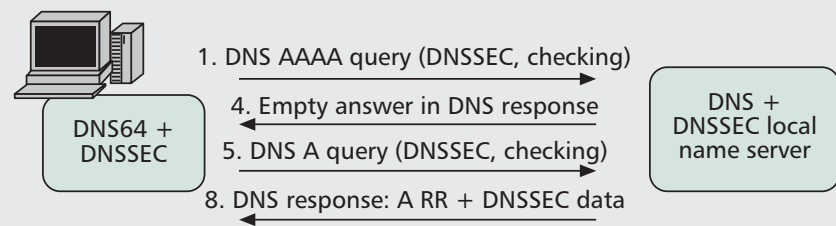

2. DNS AAAA query (DNSSEC, checking)

IPv4 DNSSEC

authoritative

3. Empty answer in DNS response

name server

6. DNS A query (DNSSEC, checking)

7. DNS response: A RR + DNSSEC data

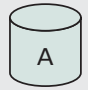

c) Validating DNSSEC-capable host

Figure 3. Different modes of DNS64 operation (detail of iterative DNS messages to the higher levels of the DNS hierarchy is not included).

is not actually performing validation but it expects the local DNS server to do it on its behalf. Typically there is a secure channel between the client and its server (e.g. protected by IPsec).

Queries from a DNSSEC-capable and validating originator (Fig. 3c). In this case, the originator asks for the DNSSEC data to perform the validation itself. Because of that, the DNS64 recursive resolver cannot send any synthetic AAAA RR in the response, or it would cause the validation to fail. This case can be handled by placing the DNS64 functionality in the client itself, after the validation module.

\section{WALKTHROUGH}

For this walkthrough we consider the topology described in Fig. 4. The NAT64 uses the WellKnown Prefix $64: f f 9 b:: / 96$ to map IPv4 addresses to IPv6, and has an IPv4 address T assigned to its IPv4 interface. The local name server implements the DNS64 function and uses the Well-Known Prefix for its synthesis. IPv6 hosts have regular stub resolvers, so they request recursive lookups to the local name server. No DNSSEC is considered.

We now describe a typical scenario in which $\mathrm{H} 1$ initiates a communication with $\mathrm{H} 2$ :

$1 \mathrm{H} 1$ performs a DNS lookup for the IPv6 address of $\mathrm{H} 2$ by sending a DNS query for a AAAA record to the local DNS/DNS64 server.

2 The local DNS/DNS64 server resolves the query, discovering that there are no AAAA records for $\mathrm{H} 2$.

3 The DNS/DNS64 server queries for a A record for $\mathrm{H} 2$, obtaining the IPv4 address $\mathrm{X}$.

4 The DNS/DNS64 server synthesizes a AAAA record by appending the IPv4 address $X$ to $64: f f 9 b:: / 96$, and includes this address in the response to $\mathrm{H} 1$.
5 After receiving the synthetic AAAA record, $\mathrm{H} 1$ sends a packet toward $\mathrm{H} 2$ from a source transport address $\left(\mathrm{Y}^{\prime}, \mathrm{Y}\right)^{3}$ to a destination transport address $(64: \mathrm{ff} 9 \mathrm{~b}: \mathrm{X}, \mathrm{x})$, where $\mathrm{y}$ and $\mathrm{x}$ are ports chosen by $\mathrm{H} 1$.

6 The packet is routed to the IPv6 interface of the NAT64 (since $64: \mathrm{ff} 9 \mathrm{~b}::$ / 96 has been associated to this interface), and the NAT64 performs the following actions:

- It selects an unused port t on its IPv4 address $\mathrm{T}$ and creates the BIB entry $\left(\mathrm{Y}^{\prime}, \mathrm{Y}\right)$ $(\mathrm{T}, \mathrm{t})$ and a session table entry ( $\mathrm{Y}^{\prime}, \mathrm{Y}$, $64: f f 9 b: x, x) \leftrightarrow(T, t, x, x)$.

- It translates the IPv6 header into an IPv4 header using stateless translation.

- It includes in the packet $(T, t)$ as source transport address and $(\mathrm{X}, \mathrm{x})$ as destination transport address, adjusting the transport layer checksum if necessary.

The NAT64 sends the translated packet to the destination $\mathrm{X}$ through the IPv4 network.

$7 \mathrm{H} 2$ node receives the packet and responds by sending a packet with destination transport address $(T, t)$ and source transport address $(\mathrm{X}, \mathrm{x})$.

8 The packet is routed to the NAT64 box, which looks for a Session table entry containing $(T, t)$. When the entry is found,

- The NAT64 translates the IPv4 header into an IPv6 header using stateless translation.

- The NAT64 includes in the packet $\left(\mathrm{Y}^{\prime}, \mathrm{Y}\right)$ as destination transport address and (Pref64:X, x) as source transport address. The translated packet is finally sent out to H1.

\section{IMPLEMENTATIONS AND EXPERIMENTS}

At the time of this writing there are already several implementations of NAT64 and DNS64. The first publicly available implementation of the tool suite was Ecdysis, ${ }^{4}$ which implements
${ }^{3}$ We use a prime (") to highlight that the address is IPv6. 


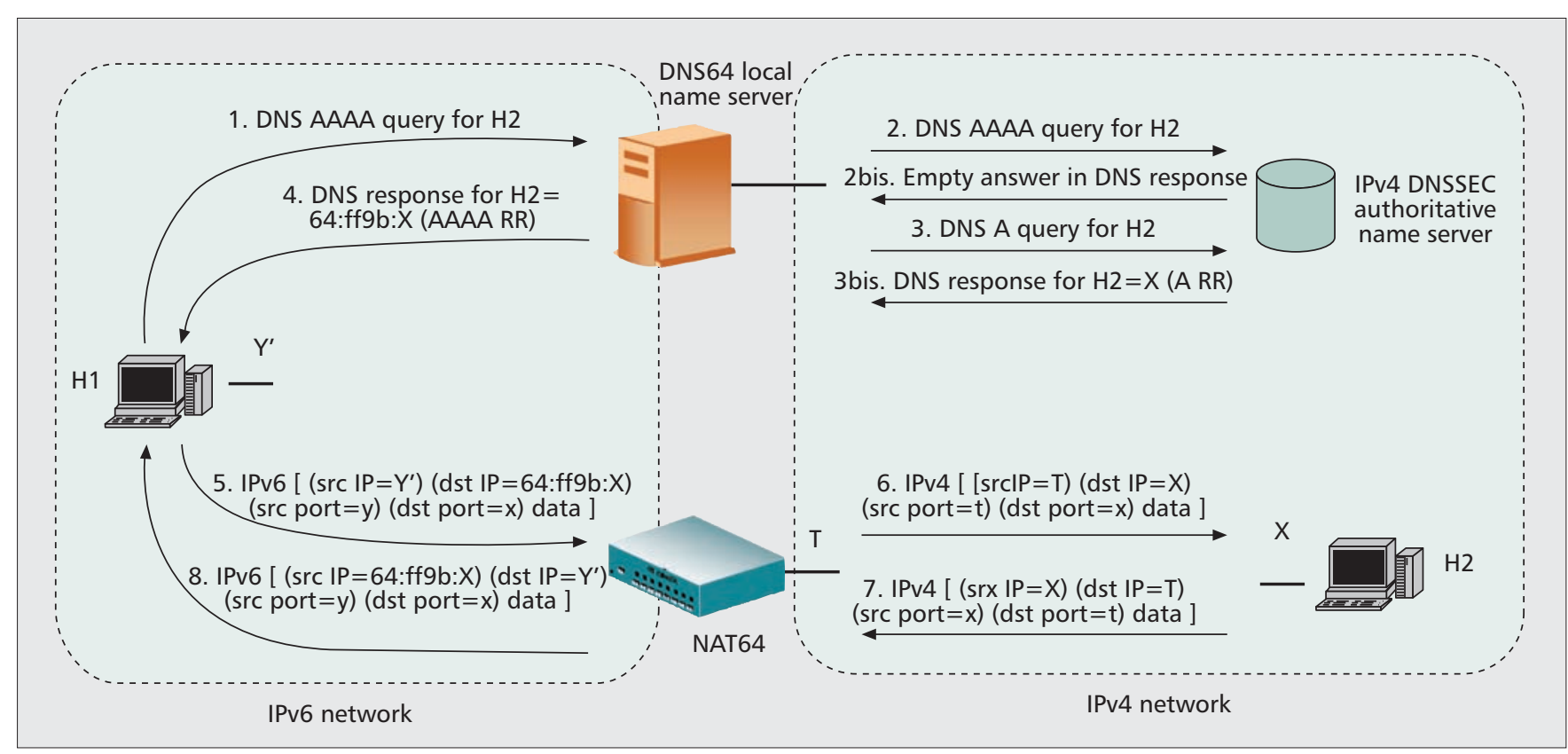

Figure 4. Walkthrough scenario (detail of iterative DNS messages to the higher levels of the DNS hierarchy is not included).

NAT64 both for Linux and BSD, and DNS64 as a patch for both BIND and Unbound. Several vendors are developing NAT64 implementations, including Juniper, Cisco, and Ericsson, some of which have already released them in commercial products. Version 9.8.0 of BIND implements DNS64.

The availability of implementations has enabled a wide range of experiments and trials from different parties, including Ericsson, China Telecom, and T-Systems.

The result of these experiences is that IPv6only networking using the NAT64/DNS64 tool suite for accessing the IPv4 Internet works well for many critical applications such as web browsing, e-mail, software upgrades, operating system services, many chat systems, and media streaming [15]. The main limitations identified by these studies include the lack of IPv6 support in applications such as several games and a very popular VoIP application, but there is not much the NAT64/DNS64 design can do about that. Another problem identified is the case where IPv4 literals are embedded in the upper layer protocols. The NAT64 does not translate those, resulting in communication failures. This can be handled by adding Application Level Gateways (ALG) in NAT64. There is ongoing work to define an ALG for FTP. Overall, NAT64/DNS64 has performed extremely well in controlled environments, such as a mobile network within which the operator selects the handsets.

\section{CONCLUSIONS}

In this article we presented the NAT64/DNS64 tool suite for IPv6 transition. NAT64 is a network address translator and protocol translator that allows communication between IPv6 and IPv4 nodes. DNS64 synthesizes AAAA RRs from the information available in A RRs for a given Fully Qualified Domain Name. This tool suite is tion in the near future. There are already several open source and commercial implementations of the tool suite.

The suite is designed to support several deployment models, although we expect two of them to be preeminent: The first one is to allow the internal nodes of an IPv6-only stub network to reach the public IPv4 Internet. In this case the NAT64/DNS64 functions can be provided either by the IPv6 stub network itself or by its direct provider, i.e. in a Carrier Grade NAT. In the second scenario, an IPv4-only stub site decides to give access to its IPv4-only servers to clients in the IPv6 Internet. For this, NAT64 can be provisioned by the IPv4 stub network. Because the DNS server of the IPv4 site is authoritative for the local data, the DNS64 function is replaced by a DNS server with AAAA RRs that contain the IPv6 representation of the IP addresses assigned to the IPv4-only servers.

As DNS64/NAT64 is a replacement for the deprecated NAT-PT, to conclude the article we compare NAT64/DNS64 and NAT-PT. In order to deal with the main limitations of NAT-PT, the DNS64/NAT64 design makes a few key architectural decisions. First, the DNS64/NAT64 manages explicitly only communications initiated from the IPv6 side. Communications initiated from the IPv4 are supported through standard NAT-traversal techniques, such as STUN and TURN, since NAT64 is designed to be NATtraversal compatible. Second, DNS64 is a fullfledged architectural component that is part of a DNS resolver. As such, it does not need to transparently intercept DNS queries. The result of these two design decisions is a more robust design, as DNS queries and data packets do not need to flow through the same path, significantly improving the reliability of the resulting network. Finally, DNS64/NAT64 uses by default the Well-Known Prefix that allows having a globally valid IPv6 representation of an IPv4 address. This implies that even if the IPv6 representation 
of an IPv4 address or the synthetic AAAA RR leak outside the realm of the NAT64, the receiving node can identify the address as being an IPv6 representation of the original IPv4 address.

\section{ACKNOWLEDGMENTS}

The research of Marcelo Bagnulo leading to these results has received funding from the Ministry of Science and Innovation of Spain, under the QUARTET project (TIN2009-13992-C0201 ) and the MEDIANET project (S2009TIC1468). The work of Alberto García-Martínez is partially supported by BONE (Building the Future Optical Network in Europe), a Network of Excellence funded by the European Commission through the 7th ICT-Framework Program, and by the MEDIANET project (S2009TIC1468).

\section{REFERENCES}

[1] E. Nordmark, "Stateless IP/ICMP Translation Algorithm (SIIT)," RFC2765, 2000.

[2] G. Tsirtsis and P. Srisuresh, "Network Address Translation - Protocol Translation (NAT-PT)," RFC2766, 2000.

[3] C. Aoun and E. Davies, "Reasons to Move the Network Address Translator - Protocol Translator (NAT-PT) to Historic Status," RFC4966, 2007.

[4] M. Bagnulo, P. Matthews, and I. van Beijnum, "Stateful NAT64: Network Address and Protocol Translation from IPv6 Clients to IPv4 Servers," RFC6146, July 2010.

[5] M. Bagnulo et al., "DNS64: DNS Extensions for Network Address Translation from IPv6 Clients to IPv4 Servers," RFC6147, July 2010.

[6] J. Rosenberg et al., "Session Traversal Utilities for NAT (STUN)," RFC5389, 2008.

[7] K. Egevang and P. Francis, "The IP Network Address Translator (NAT)," RFC1631, 1994.

[8] T. Hain, "Architectural Implications of NAT," RFC2993, 2000.

[9] S. Guha, Eds. et al., "NAT Behavioral Requirements for TCP," RFC5382, 2008
[10] F. Audet, Ed., and C. Jennings, "Network Address Translation (NAT) Behavioral Requirements for Unicast UDP," RFC4787, 2007.

[11] P. Srisuresh et al., "NAT Behavioral Requirements for ICMP," RFC5508, 2009.

[12] R. Mahy, P. Matthews, and J. Rosenberg, "Traversal Using Relays around NAT (TURN): Relay Extensions to Session Traversal Utilities for NAT (STUN)," RFC 5766, 2010.

[13] X. Li, C. Bao, and F. Baker, "IP/ICMP Translation Algorithm," RFC6145, 2010.

[14] C. Bao et al., "IPv6 Addressing of IPv4/IPv6 Translators," RFC6052, 2010.

[15] J. Arkko and A. Keranen, "Experiences from an IPv6Only Network," RFC6586, 2012.

\section{BIOGRAPHIES}

MARCELo BAGNulo (marcelo@it.uc3m.es) received an Electrical Engineering degree in 1999 from the University of Uruguay, and a Ph.D. in Telecommunications in 2005 from the University Carlos III of Madrid, Spain. In 2000 he joined the University Carlos III of Madrid (UC3M), where he has been an associate professor since 2006 . He has published several papers in technical journals, magazines, and conferences. His main interest areas are IPv6 and interdomain routing.

Alberto GARCíA-MARTínez (alberto@it.uc3m.es) received a Telecommunications Engineering degree in 1995 and a Ph. D in Telecommunications in 1999 both from the UPM Spain. In 1998 he joined the University Carlos III of Madrid (UC3M), where he has been an associate professor since 2001. His main interest areas are IPv6 and routing.

ILJITSCH VAN BEIJNUM (iljitsch.vanbeijnum@imdea.org) received the Bachelor of Information and Communication Technology degree from the Haagse Hogeschool in The Hague in 2005 and the Master of Telematics Engineering degree from UC3M in Madrid in 2008. He has worked in the internet service provider business since 1995 and wrote books about BGP and IPv6 and many articles in the trade press. He currently works for Institute IMDEA Networks as a research assistant, pursuing the Ph.D. degree at UC3M.
The result of these two design decisions is a more robust design, as DNS queries and data packets do not need to flow through the same path, significantly improving the reliability of the resulting network. 